\title{
Constituent Parsing by Classification
}

\author{
Joseph Turian and I. Dan Melamed \\ \{lastname\}@cs.nyu.edu \\ Computer Science Department \\ New York University \\ New York, New York 10003
}

\begin{abstract}
Ordinary classification techniques can drive a conceptually simple constituent parser that achieves near state-of-the-art accuracy on standard test sets. Here we present such a parser, which avoids some of the limitations of other discriminative parsers. In particular, it does not place any restrictions upon which types of features are allowed. We also present several innovations for faster training of discriminative parsers: we show how training can be parallelized, how examples can be generated prior to training without a working parser, and how independently trained sub-classifiers that have never done any parsing can be effectively combined into a working parser. Finally, we propose a new figure-of-merit for bestfirst parsing with confidence-rated inferences. Our implementation is freely available at: http: //cs.nyu.edu/ turian/ software/parser/
\end{abstract}

\section{Introduction}

Discriminative machine learning methods have improved accuracy on many NLP tasks, such as POStagging (Toutanova et al., 2003), machine translation (Och \& Ney, 2002), and relation extraction (Zhao \& Grishman, 2005). There are strong reasons to believe the same would be true of parsing. However, only limited advances have been made thus far, perhaps due to various limitations of extant discriminative parsers. In this paper, we present some innovations aimed at reducing or eliminating some of these limitations, specifically for the task of constituent parsing:

- We show how constituent parsing can be performed using standard classification techniques.

- Classifiers for different non-terminal labels can be induced independently and hence training can be parallelized.

- The parser can use arbitrary information to evaluate candidate constituency inferences.

- Arbitrary confidence scores can be aggregated in a principled manner, which allows beam search.

In Section 2 we describe our approach to parsing. In Section 3 we present experimental results.

The following terms will help to explain our work. A span is a range over contiguous words in the input sentence. Spans cross if they overlap but neither contains the other. An item (or constituent) is a (span, label) pair. A state is a set of parse items, none of which may cross. A parse inference is a pair $(S, i)$, given by the current state $S$ and an item $i$ to be added to it. A parse path (or history) is a sequence of parse inferences over some input sentence (Klein \& Manning, 2001). An item ordering (ordering, for short) constrains the order in which items may be inferred. In particular, if we prescribe a complete item ordering, the parser is deterministic (Marcus, 1980) and each state corresponds to a unique parse path. For some input sentence and gold-standard parse, a state is correct if the parser can infer zero or more additional items to obtain the gold-standard parse. A parse path is correct if it leads to a correct state. An 
inference is correct if adding its item to its state is correct.

\section{Parsing by Classification}

Recall that with typical probabilistic parsers, our goal is to output the parse $\hat{P}$ with the highest likelihood for the given input sentence $x$ :

$$
\begin{aligned}
\hat{P} & =\underset{P \in \mathbf{P}(x)}{\arg \max } \operatorname{Pr}(P) \\
& =\underset{P \in \mathbf{P}(x)}{\arg \max } \prod_{I \in P} \operatorname{Pr}(I)
\end{aligned}
$$

or, equivalently,

$$
=\underset{P \in \mathbf{P}(x)}{\arg \max } \sum_{I \in P} \log (\operatorname{Pr}(I))
$$

where each $I$ is a constituency inference in the parse path $P$.

In this work, we explore a generalization in which each inference $I$ is assigned a real-valued confidence score $Q(I)$ and individual confidences are aggregated using some function $\mathcal{A}$, which need not be a sum or product:

$$
\hat{P}=\underset{P \in \mathbf{P}(x)}{\arg \max } \underset{I \in P}{\mathcal{A}} Q(I)
$$

In Section 2.1 we describe how we induce scoring function $Q(I)$. In Section 2.2 we discuss the aggregation function $\mathcal{A}$. In Section 2.3 we describe the method used to restrict the size of the search space over $\mathbf{P}(x)$.

\subsection{Learning the Scoring Function $Q(I)$}

During training, our goal is to induce the scoring function $Q$, which assigns a real-valued confidence score $Q(I)$ to each candidate inference $I$ (Equation 4). We treat this as a classification task: If inference $I$ is correct, we would like $Q(I)$ to be a positive value, and if inference $I$ is incorrect, we would like $Q(I)$ to be a negative value.

Training discriminative parsers can be computationally very expensive. Instead of having a single classifier score every inference, we parallelize training by inducing 26 sub-classifiers, one for each constituent label $\lambda$ in the Penn Treebank (Taylor, Marcus, \& Santorini, 2003): $Q\left(I_{\lambda}\right)=Q_{\lambda}\left(I_{\lambda}\right)$, where $Q_{\lambda}$ is the $\lambda$-classifier and $I_{\lambda}$ is an inference that infers a constituent with label $\lambda$. For example, the VPclassifier $Q_{\text {VP }}$ would score the VP-inference in Figure 1 , preferably assigning it a positive confidence.
Figure 1 A candidate VP-inference, with headchildren annotated using the rules given in (Collins, 1999).

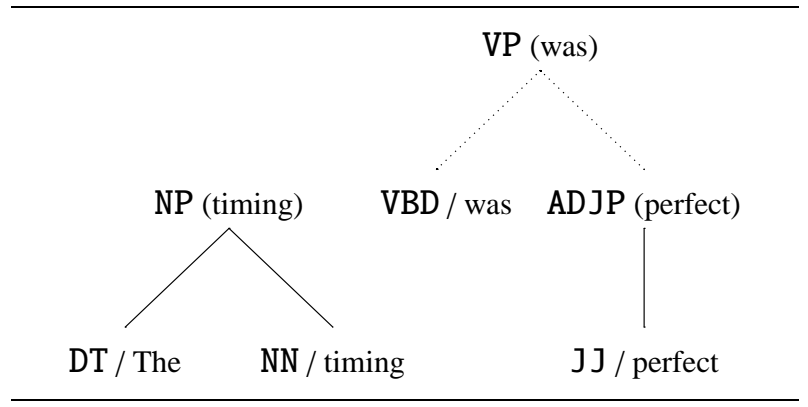

Each $\lambda$-classifier is independently trained on training set $E_{\lambda}$, where each example $e_{\lambda} \in E_{\lambda}$ is a tuple $\left(I_{\lambda}, y\right)$, $I_{\lambda}$ is a candidate $\lambda$-inference, and $y \in\{ \pm 1\} . y=+1$ if $I_{\lambda}$ is a correct inference and -1 otherwise. This approach differs from that of Yamada and Matsumoto (2003) and Sagae and Lavie (2005), who parallelize according to the POS tag of one of the child items.

\subsubsection{Generating Training Examples}

Our method of generating training examples does not require a working parser, and can be run prior to any training. It is similar to the method used in the literature by deterministic parsers (Yamada \& Matsumoto, 2003; Sagae \& Lavie, 2005) with one exception: Depending upon the order constituents are inferred, there may be multiple bottom-up paths that lead to the same final parse, so to generate training examples we choose a single random path that leads to the gold-standard parse tree. ${ }^{1}$ The training examples correspond to all candidate inferences considered in every state along this path, nearly all of which are incorrect inferences (with $y=-1$ ). For instance, only $4.4 \%$ of candidate NP-inferences are correct.

\subsubsection{Training Algorithm}

During training, for each label $\lambda$ we induce scoring function $Q_{\lambda}$ to minimize the loss over training examples $E_{\lambda}$ :

$$
Q_{\lambda}=\underset{Q_{\lambda}^{\prime}}{\arg \min } \sum_{\left(I_{\lambda}, y\right) \in E_{\lambda}} L\left(y \cdot Q_{\lambda}^{\prime}\left(I_{\lambda}\right)\right)
$$

\footnotetext{
1 The particular training tree paths used in our experiments are included in the aforementioned implementation so that our results can be replicated under the same experimental conditions.
} 
where $y \cdot Q_{\lambda}\left(I_{\lambda}\right)$ is the margin of example $\left(I_{\lambda}, y\right)$. Hence, the learning task is to maximize the margins of the training examples, i.e. induce scoring function $Q_{\lambda}$ such that it classifies correct inferences with positive confidence and incorrect inferences with negative confidence. In our work, we minimized the logistic loss:

$$
L(z)=\log (1+\exp (-z))
$$

i.e. the negative log-likelihood of the training sample.

Our classifiers are ensembles of decisions trees, which we boost (Schapire \& Singer, 1999) to minimize the above loss using the update equations given in Collins, Schapire, and Singer (2002). More specifically, classifier $Q_{\lambda}^{T}$ is an ensemble comprising decision trees $q_{\lambda}^{1}, \ldots, q_{\lambda}^{T}$, where:

$$
Q_{\lambda}^{T}\left(I_{\lambda}\right)=\sum_{t=1}^{T} q_{\lambda}^{t}\left(I_{\lambda}\right)
$$

At iteration $t$, decision tree $q_{\lambda}^{t}$ is grown, its leaves are confidence-rated, and it is added to the ensemble. The classifier for each constituent label is trained independently, so we henceforth omit $\lambda$ subscripts.

An example $(I, y)$ is assigned weight $w^{t}(I, y):^{2}$

$$
w^{t}(I, y)=\frac{1}{1+\exp \left(y \cdot Q^{t-1}(I)\right)}
$$

The total weight of $y$-value examples that fall in leaf $f$ is $W_{f, y}^{t}$ :

$$
W_{f, y}^{t}=\sum_{\substack{\left(I, y^{\prime}\right) \in E \\ y^{\prime}=y, I \in f}} w^{t}(I, y)
$$

and this leaf has $\operatorname{loss} Z_{f}^{t}$ :

$$
Z_{f}^{t}=2 \cdot \sqrt{W_{f,+}^{t} \cdot W_{f,-}^{t}}
$$

Growing the decision tree: The loss of the entire decision tree $q^{t}$ is

$$
Z\left(q^{t}\right)=\sum_{\text {leaf } f \in q^{t}} Z_{f}^{t}
$$

2 If we were to replace this equation with $w^{t}(I, y)=$ $\exp \left(y \cdot Q^{t-1}(I)\right)^{-1}$, but leave the remainder of the algorithm unchanged, this algorithm would be confi dence-rated AdaBoost (Schapire \& Singer, 1999), minimizing the exponential loss $L(z)=\exp (-z)$. In preliminary experiments, however, we found that the logistic loss provided superior generalization accuracy.
We will use $Z^{t}$ as a shorthand for $Z\left(q^{t}\right)$. When growing the decision tree, we greedily choose node splits to minimize this $Z$ (Kearns \& Mansour, 1999). In particular, the loss reduction of splitting leaf $f$ using feature $\phi$ into two children, $f \wedge \phi$ and $f \wedge \neg \phi$, is $\Delta Z_{f}^{t}(\phi)$ :

$$
\Delta Z_{f}^{t}(\phi)=Z_{f}^{t}-\left(Z_{f \wedge \phi}^{t}+Z_{f \wedge \neg \phi}^{t}\right)
$$

To split node $f$, we choose the $\hat{\phi}$ that reduces loss the most:

$$
\hat{\phi}=\underset{\phi \in \Phi}{\arg \max } \Delta Z_{f}^{t}(\phi)
$$

Confidence-rating the leaves: Each leaf $f$ is confidence-rated as $\kappa_{f}^{t}$ :

$$
\kappa_{f}^{t}=\frac{1}{2} \cdot \log \frac{W_{f,+}^{t}+\epsilon}{W_{f,-}^{t}+\epsilon}
$$

Equation 14 is smoothed by the $\epsilon$ term (Schapire \& Singer, 1999) to prevent numerical instability in the case that either $W_{f,+}^{t}$ or $W_{f,-}^{t}$ is 0 . In our experiments, we used $\epsilon=10^{-8}$. Although our example weights are unnormalized, so far we've found no benefit from scaling $\epsilon$ as Collins and Koo (2005) suggest. All inferences that fall in a particular leaf node are assigned the same confidence: if inference $I$ falls in leaf node $f$ in the $t$ th decision tree, then $q^{t}(I)=\kappa_{f}^{t}$.

\subsubsection{Calibrating the Sub-Classifiers}

An important concern is when to stop growing the decision tree. We propose the minimum reduction in loss (MRL) stopping criterion: During training, there is a value $\Theta^{t}$ at iteration $t$ which serves as a threshold on the minimum reduction in loss for leaf splits. If there is no splitting feature for leaf $f$ that reduces loss by at least $\Theta^{t}$ then $f$ is not split. Formally, leaf $f$ will not be bisected during iteration $t$ if $\max _{\phi \in \Phi} \Delta Z_{f}^{t}(\phi)<\Theta^{t}$. The MRL stopping criterion is essentially $\ell_{0}$ regularization: $\Theta^{t}$ corresponds to the $\ell_{0}$ penalty parameter and each feature with non-zero confidence incurs a penalty of $\Theta^{t}$, so to outweigh the penalty each split must reduce loss by at least $\Theta^{t}$.

$\Theta^{t}$ decreases monotonically during training at the slowest rate possible that still allows training to proceed. We start by initializing $\Theta^{1}$ to $\infty$, and at the beginning of iteration $t$ we decrease $\Theta^{t}$ only if the root node $\emptyset$ of the decision tree cannot be split. Otherwise, $\Theta^{t}$ is set to $\Theta^{t-1}$. Formally, 
$\Theta^{t}=\min \left(\Theta^{t-1}, \max _{\phi \in \Phi} \Delta Z_{\emptyset}^{t}(\phi)\right)$. In this manner, the decision trees are induced in order of decreasing $\Theta^{t}$.

During training, the constituent classifiers $Q_{\lambda}$ never do any parsing per se, and they train at different rates: If $\lambda \neq \lambda^{\prime}$, then $\Theta_{\lambda}^{t}$ isn't necessarily equal to $\Theta_{\lambda^{\prime}}^{t}$. We calibrate the different classifiers by picking some meta-parameter $\hat{\Theta}$ and insisting that the sub-classifiers comprised by a particular parser have all reached some fixed $\Theta$ in training. Given $\hat{\Theta}$, the constituent classifier for label $\lambda$ is $Q_{\lambda}^{t}$, where $\Theta_{\lambda}^{t} \geq \hat{\Theta}>\Theta_{\lambda}^{t+1}$. To obtain the final parser, we cross-validate $\hat{\Theta}$, picking the value whose set of constituent classifiers maximizes accuracy on a development set.

\subsubsection{Types of Features used by the Scoring Function}

Our parser operates bottom-up. Let the frontier of a state be the top-most items (i.e. the items with no parents). The children of a candidate inference are those frontier items below the item to be inferred, the left context items are those frontier items to the left of the children, and the right context items are those frontier items to the right of the children. For example, in the candidate VP-inference shown in Figure 1, the frontier comprises the NP, VBD, and ADJP items, the VBD and ADJP items are the children of the VPinference (the VBD is its head child), the NP is the left context item, and there are no right context items.

The design of some parsers in the literature restricts the kinds of features that can be usefully and efficiently evaluated. Our scoring function and parsing algorithm have no such limitations. $Q$ can, in principle, use arbitrary information from the history to evaluate constituent inferences. Although some of our feature types are based on prior work (Collins, 1999; Klein \& Manning, 2003; Bikel, 2004), we note that our scoring function uses more history information than typical parsers.

All features check whether an item has some property; specifically, whether the item's label/headtag/headword is a certain value. These features perform binary tests on the state directly, unlike Henderson (2003) which works with an intermediate representation of the history. In our baseline setup, feature set $\Phi$ contained five different feature types, described in Table 1.
Table 2 Feature item groups.

- all children

- all non-head children

- all non-leftmost children

- all non-rightmost children

- all children left of the head

- all children right of the head

- head-child and all children left of the head

- head-child and all children right of the head

\subsection{Aggregating Confidences}

To get the cumulative score of a parse path $P$, we apply aggregator $\mathcal{A}$ over the confidences $Q(I)$ in Equation 4 . Initially, we defined $\mathcal{A}$ in the customary fashion as summing the loss of each inference's confidence:

$$
\hat{P}=\underset{P \in \mathbf{P}(x)}{\arg \max }\left(-\sum_{I \in P} L(Q(I))\right)
$$

with the logistic loss $L$ as defined in Equation 6. (We negate the final sum because we want to minimize the loss.) This definition of $\mathcal{A}$ is motivated by viewing $L$ as a negative log-likelihood given by a logistic function (Collins et al., 2002), and then using Equation 3. It is also inspired by the multiclass loss-based decoding method of Schapire and Singer (1999). With this additive aggregator, loss monotonically increases as inferences are added, as in a PCFG-based parser in which all productions decrease the cumulative probability of the parse tree.

In preliminary experiments, this aggregator gave disappointing results: precision increased slightly, but recall dropped sharply. Exploratory data analysis revealed that, because each inference incurs some positive loss, the aggregator very cautiously builds the smallest trees possible, thus harming recall. We had more success by defining $\mathcal{A}$ to maximize the minimum confidence. Essentially,

$$
\hat{P}=\underset{P \in \mathbf{P}(x)}{\arg \max } \min _{I \in P} Q(I)
$$

Ties are broken according to the second lowest confidence, then the third lowest, and so on.

\subsection{Search}

Given input sentence $x$, we choose the parse path $P$ in $\mathbf{P}(x)$ with the maximum aggregated score (Equation 4). Since it is computationally intractable to 
Table 1 Types of features.

- Child item features test if a particular child item has some property. E.g. does the item one right of the head have headword "perfect"? (True in Figure 1)

- Context item features test if a particular context item has some property. E.g. does the first item of left context have headtag NN? (True)

- Grandchild item features test if a particular grandchild item has some property. E.g. does the leftmost child of the rightmost child item have label $\mathrm{J} J$ ? (True)

- Exists features test if a particular group of items contains an item with some property. E.g. does some non-head child item have label ADJP? (True) Exists features select one of the groups of items specified in Table 2. Alternately, they can select the terminals dominated by that group. E.g. is there some terminal item dominated by non-rightmost children items that has headword "quux"? (False)

consider every possible sequence of inferences, we use beam search to restrict the size of $\mathbf{P}(x)$. As an additional guard against excessive computation, search stopped if more than a fixed maximum number of states were popped from the agenda. As usual, search also ended if the highest-priority state in the agenda could not have a better aggregated score than the best final parse found thus far.

\section{Experiments}

Following Taskar, Klein, Collins, Koller, and Manning (2004), we trained and tested on $\leq 15$ word sentences in the English Penn Treebank (Taylor et al., 2003), $10 \%$ of the entire treebank by word count. ${ }^{3}$ We used sections 02-21 (9753 sentences) for training, section 24 (321 sentences) for development, and section 23 (603 sentences) for testing, preprocessed as per Table 3. We evaluated our parser using the standard PARSEVAL measures (Black et al., 1991): labelled precision, recall, and F-measure (LPRC, LRCL, and LFMS, respectively), which are computed based on the number of constituents in the parser's output that match those in the gold-standard parse. We tested whether the observed differences in PARSEVAL measures are significant at $p=0.05$ using a stratified shuffling test (Cohen, 1995, Section 5.3.2) with one million trials. ${ }^{4}$

As mentioned in Section 1, the parser cannot infer any item that crosses an item already in the state.

3 There was insufficient time before deadline to train on all sentences.

4 The shuffling test we used was originally implemented by Dan Bikel (http://www.cis.upenn.edu/ dbikel/ software.html) and subsequently modifi ed to compute $p$ values for LFMS differences.
We placed three additional candidacy restrictions on inferences: (a) Items must be inferred under the bottom-up item ordering; (b) To ensure the parser does not enter an infinite loop, no two items in a state can have both the same span and the same label; (c) An item can have no more than $K=5$ children. (Only $0.24 \%$ of non-terminals in the preprocessed development set have more than five children.) The number of candidate inferences at each state, as well as the number of training examples generated by the algorithm in Section 2.1.1, is proportional to $K$. In our experiment, there were roughly $\left|E_{\lambda}\right| \approx 1.7$ million training examples for each classifier.

\subsection{Baseline}

In the baseline setting, context item features (Section 2.1.4) could refer to the two nearest items of context in each direction. The parser used a beam width of 1000, and was terminated in the rare event that more than 10,000 states were popped from the agenda. Figure 2 shows the accuracy of the baseline on the development set as training progresses. Cross-validating the choice of $\hat{\Theta}$ against the LFMS (Section 2.1.3) suggested an optimum of $\hat{\Theta}=1.42$. At this $\hat{\Theta}$, there were a total of 9297 decision tree splits in the parser (summed over all constituent classifiers), $\mathrm{LFMS}=87.16, \mathrm{LRCL}=86.32$, and $\mathrm{LPRC}=88.02$.

\subsection{Beam Width}

To determine the effect of the beam width on the accuracy, we evaluated the baseline on the development set using a beam width of 1, i.e. parsing entirely greedily (Wong \& Wu, 1999; Kalt, 2004; Sagae \& Lavie, 2005). Table 4 compares the base- 
Table 3 Steps for preprocessing the data. Starred steps are performed only on input with tree structure.

1. * Strip functional tags and trace indices, and remove traces.

2. * Convert PRT to ADVP. (This convention was established by Magerman (1995).)

3. Remove quotation marks (i.e. terminal items tagged “ ' or ' '). (Bikel, 2004)

4. * Raise punctuation. (Bikel, 2004)

5. Remove outermost punctuation. ${ }^{a}$

6. * Remove unary projections to self (i.e. duplicate items with the same span and label).

7. POS tag the text using Ratnaparkhi (1996).

8. Lowercase headwords.

9. Replace any word observed fewer than 5 times in the (lower-cased) training sentences with UNK.

a As pointed out by an anonymous reviewer of Collins (2003), removing outermost punctuation may discard useful information. It's also worth noting that Collins and Roark (2004) saw a LFMS improvement of $0.8 \%$ over their baseline discriminative parser after adding punctuation features, one of which encoded the sentence-fi nal punctuation.

Figure 2 PARSEVAL scores of the baseline on the $\leq 15$ words development set of the Penn Treebank. The top $x$-axis shows accuracy as the minimum reduction in loss $\hat{\Theta}$ decreases. The bottom shows the corresponding number of decision tree splits in the parser, summed over all classifiers.

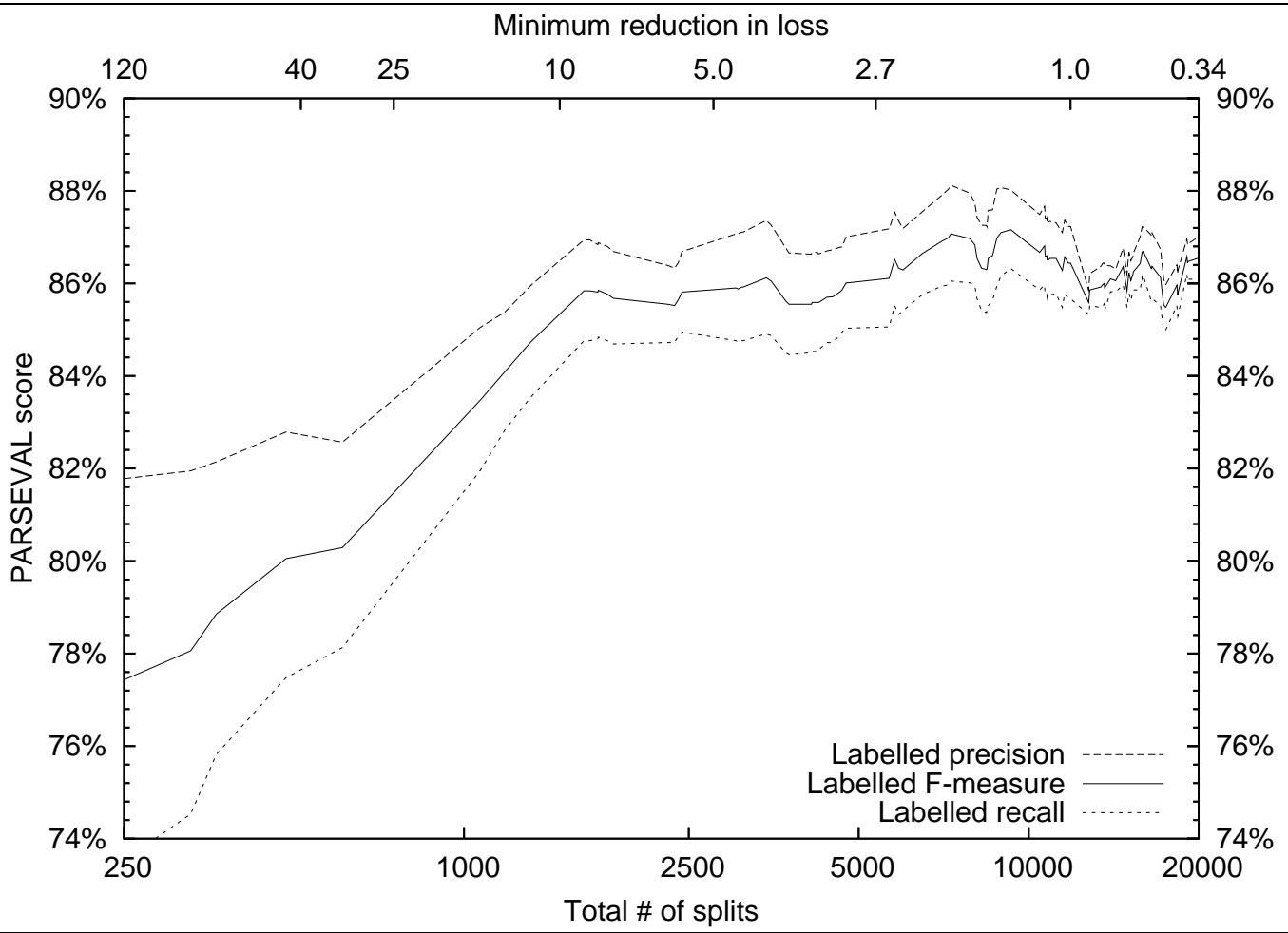

line results on the development set with a beam width of 1 and a beam width of $1000 .^{5}$ The wider beam seems to improve the PARSEVAL scores of the parser, although we were unable to detect a statistically significant improvement in LFMS on our relatively small development set.

5 Using a beam width of 100,000 yielded output identical to using a beam width of 1000 .

\subsection{Context Size}

Table 5 compares the baseline to parsers that could not examine as many context items. A significant portion of the baseline's accuracy is due to contextual clues, as evidenced by the poor accuracy of the no context run. However, we did not detect a significant difference between using one context item or two. 


\begin{tabular}{|c|c|c|c|c|}
\hline \multicolumn{5}{|c|}{$\begin{array}{l}\text { Table } 4 \text { PARSEVAL results on the } \leq 15 \text { words } \\
\text { development set of the baseline, varying the beam } \\
\text { width. Also, the MRL that achieved this LFMS and } \\
\text { the total number of decision tree splits at this MRL. }\end{array}$} \\
\hline & $\begin{array}{c}\text { Dev } \\
\text { LFMS }\end{array}$ & $\begin{array}{c}\text { Dev } \\
\text { LRCL }\end{array}$ & $\begin{array}{l}\text { Dev } \\
\text { LPRC }\end{array}$ & $\begin{array}{c}\text { MRL \#splits } \\
\hat{\Theta} \text { total }\end{array}$ \\
\hline $\mathrm{Be}$ & 86.36 & 86.20 & 86.53 & $2.03 \quad 7068$ \\
\hline Baseline & 87.16 & 86.32 & 88.02 & 1.429297 \\
\hline
\end{tabular}

Table 5 PARSEVAL results on the $\leq 15$ words development set, given the amount of context available. is statistically significant. The score differences between "context 0 " and "context 1 " are significant, whereas the differences between "context 1 " and the baseline are not.

\begin{tabular}{|c|c|c|c|c|}
\hline & $\begin{array}{c}\text { Dev } \\
\text { LFMS }\end{array}$ & $\begin{array}{c}\text { Dev } \\
\text { LRCL }\end{array}$ & $\begin{array}{c}\text { Dev } \\
\text { LPRC }\end{array}$ & $\begin{array}{cc}\text { MRL } & \text { \#splits } \\
\hat{\Theta} & \text { total }\end{array}$ \\
\hline Context 0 & 75.15 & 75.28 & 75.03 & $3.38 \quad 3815$ \\
\hline Context 1 & 86.93 & 85.78 & 88.12 & $2.45 \quad 5588$ \\
\hline Baseline & 87.16 & 86.32 & 88.02 & 1.429297 \\
\hline
\end{tabular}

Table 6 PARSEVAL results of decision stumps on the $\leq 15$ words development set, through 8200 splits. The differences between the stumps run and the baseline are statistically significant.

\begin{tabular}{|c|c|c|c|c|}
\hline & $\begin{array}{c}\text { Dev } \\
\text { LFMS }\end{array}$ & $\begin{array}{c}\text { Dev } \\
\text { LRCL }\end{array}$ & $\begin{array}{c}\text { Dev } \\
\text { LPRC }\end{array}$ & $\begin{array}{c}\text { MRL \#splits } \\
\hat{\Theta} \text { total }\end{array}$ \\
\hline Stumps & 85.72 & 84.65 & 86.82 & $2.39 \quad 5217$ \\
\hline Baseline & 87.07 & 86.05 & 88.12 & 1.927283 \\
\hline
\end{tabular}

\subsection{Decision Stumps}

Our features are of relatively fine granularity. To test if a less powerful machine could provide accuracy comparable to the baseline, we trained a parser in which we boosted decisions stumps, i.e. decision trees of depth 1. Stumps are equivalent to learning a linear discriminant over the atomic features. Since the stumps run trained quite slowly, it only reached 8200 splits total. To ensure a fair comparison, in Table 6 we chose the best baseline parser with at most 8200 splits. The LFMS of the stumps run on the development set was $85.72 \%$, significantly less accurate than the baseline.

For example, Figure 3 shows a case where NP classification better served by the informative conjunction $\phi_{1} \wedge \phi_{2}$ found by the decision trees. Given
Figure 3 An example of a decision (a) stump and (b) tree for scoring NP-inferences. Each leaf's value is the confidence assigned to all inferences that fall in this leaf. $\phi_{1}$ asks "does the first child have a determiner headtag?". $\phi_{2}$ asks "does the last child have a noun label?". NP classification is better served by the informative conjunction $\phi_{1} \wedge \phi_{2}$ found by the decision trees. (a)

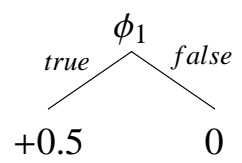

(b)

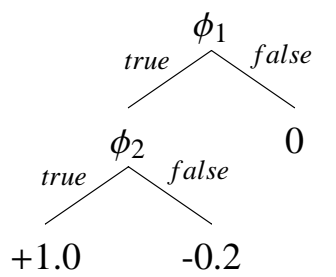

Table 7 PARSEVAL results of deterministic parsers on the $\leq 15$ words development set through 8700 splits. A shaded cell means that the difference between this value and that of the baseline is statistically significant. All differences between $12 \mathrm{r}$ and $\mathrm{r} 2 \mathrm{l}$ are significant.

\begin{tabular}{|c|c|c|c|c|}
\hline & $\begin{array}{c}\text { Dev } \\
\text { LFMS }\end{array}$ & $\begin{array}{c}\text { Dev } \\
\text { LRCL }\end{array}$ & $\begin{array}{c}\text { Dev } \\
\text { LPRC }\end{array}$ & $\begin{array}{c}\text { MRL \#splits } \\
\hat{\Theta} \text { total }\end{array}$ \\
\hline $12 r$ & 83.61 & 82.71 & 84.54 & $3.37 \quad 2157$ \\
\hline $\mathrm{r} 21$ & 85.76 & 85.37 & 86.15 & 3.391881 \\
\hline Baseline & 87.07 & 86.05 & 88.12 & 1.927283 \\
\hline
\end{tabular}

the sentence "The man left", at the initial state there are six candidate NP-inferences, one for each span, and "(NP The man)" is the only candidate inference that is correct. $\phi_{1}$ is true for the correct inference and two of the incorrect inferences ("(NP The)" and "(NP The man left)"). $\phi_{1} \wedge \phi_{2}$, on the other hand, is true only for the correct inference, and so it is better at discriminating NPs over this sample.

\subsection{Deterministic Parsing}

Our baseline parser simulates a non-deterministic machine, as at any state there may be several correct decisions. We trained deterministic variations of the parser, for which we imposed strict left-to-right (12r) and right-to-left (r2l) item orderings. For these variations we generated training examples using the corresponding unique path to each gold-standard training tree. The r21 run reached only 8700 splits total, so in Table 7 we chose the best baseline and 12r 


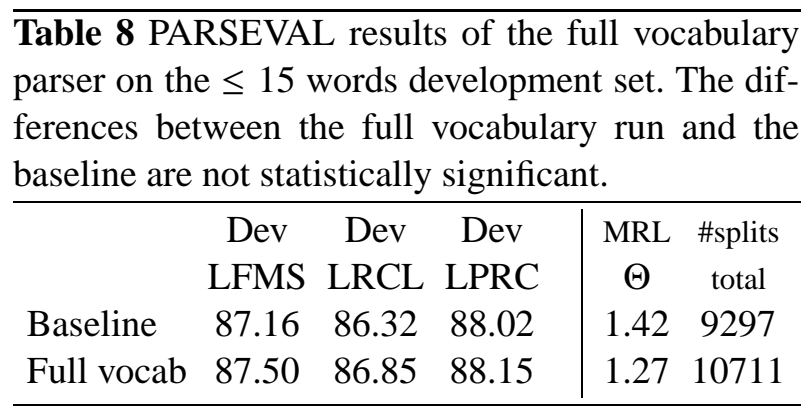

parser with at most 8700 splits.

r2l parsing is significantly more accurate than $12 \mathrm{r}$. The reason is that the deterministic runs ( $12 \mathrm{r}$ and $\mathrm{r} 2 \mathrm{l}$ ) must avoid prematurely inferring items that come later in the item ordering. This puts the $12 \mathrm{r}$ parser in a tough spot. If it makes far-right decisions, it's more likely to prevent correct subsequent decisions that are earlier in the $12 \mathrm{r}$ ordering, i.e. to the left. But if it makes far-left decisions, then it goes against the right-branching tendency of English sentences. In contrast, the $\mathrm{r} 21$ parser is more likely to be correct when it infers far-right constituents.

We also observed that the accuracy of the deterministic parsers dropped sharply as training progressed (See Figure 4). This behavior was unexpected, as the accuracy curve levelled off in every other experiment. In fact, the accuracy of the deterministic parsers fell even when parsing the training data. To explain this behavior, we examined the margin distributions of the r2l NP-classifier (Figure 5). As training progressed, the NP-classifier was able to reduce loss by driving up the margins of the incorrect training examples, at the expense of incorrectly classifying a slightly increased number of correct training examples. However, this is detrimental to parsing accuracy. The more correct inferences with negative confidence, the less likely it is at some state that the highest confidence inference is correct. This effect is particularly pronounced in the deterministic setting, where there is only one correct inference per state.

\subsection{Full Vocabulary}

As in traditional parsers, the baseline was smoothed by replacing any word that occurs fewer than five times in the training data with the special token UNK (Table 3.9). Table 8 compares the baseline to a full vocabulary run, in which the vocabulary contained all words observed in the training data. As evidenced by the results therein, controlling for lexical sparsity did not significantly improve accuracy in our setting. In fact, the full vocabulary run is slightly more accurate than the baseline on the development set, although this difference was not statistically significant. This was a late-breaking result, and we used the full vocabulary condition as our final parser for parsing the test set.

\subsection{Test Set Results}

Table 9 shows the results of our best parser on the $\leq 15$ words test set, as well as the accuracy reported for a recent discriminative parser (Taskar et al., 2004) and scores we obtained by training and testing the parsers of Charniak (2000) and Bikel (2004) on the same data. Bikel (2004) is a "clean room" reimplementation of the Collins parser (Collins, 1999) with comparable accuracy. Both Charniak (2000) and Bikel (2004) were trained using the goldstandard tags, as this produced higher accuracy on the development set than using Ratnaparkhi (1996)'s tags.

\subsection{Exploratory Data Analysis}

To gain a better understanding of the weaknesses of our parser, we examined a sample of 50 development sentences that the full vocabulary parser did not get entirely correct. Besides noise and cases of genuine ambiguity, the following list outlines all error types that occurred in more than five sentences, in roughly decreasing order of frequency. (Note that there is some overlap between these groups.)

- ADVPs and ADJPs A disproportionate amount of the parser's error was due to ADJPs and ADVPs. Out of the $12.5 \%$ total error of the parser on the development set, an absolute $1.0 \%$ was due to ADVPs, and $0.9 \%$ due to ADJPs. The parser had LFMS $=78.9 \%$, LPRC $=82.5 \%, \mathrm{LRCL}=75.6 \%$ on ADVPs, and LFMS $=68.0 \%$, LPRC $=$ $71.2 \%, \mathrm{LRCL}=65.0 \%$ on $\mathrm{ADJPs}$.

These constructions can sometimes involve tricky attachment decisions. For example, in the fragment "to get fat in times of crisis", the parser's output was "(VP to (VP get (ADJP fat (PP in (NP (NP times) (PP of (NP crisis)))))))" instead of the correct construction "(VP to (VP get (ADJP fat) (PP in (NP (NP times) (PP of (NP crisis))))))". 
Figure 4 LFMS of the baseline and the deterministic runs on the $\leq 15$ words development set of the Penn Treebank. The $x$-axis shows the LFMS as training progresses and the number of decision tree splits increases.

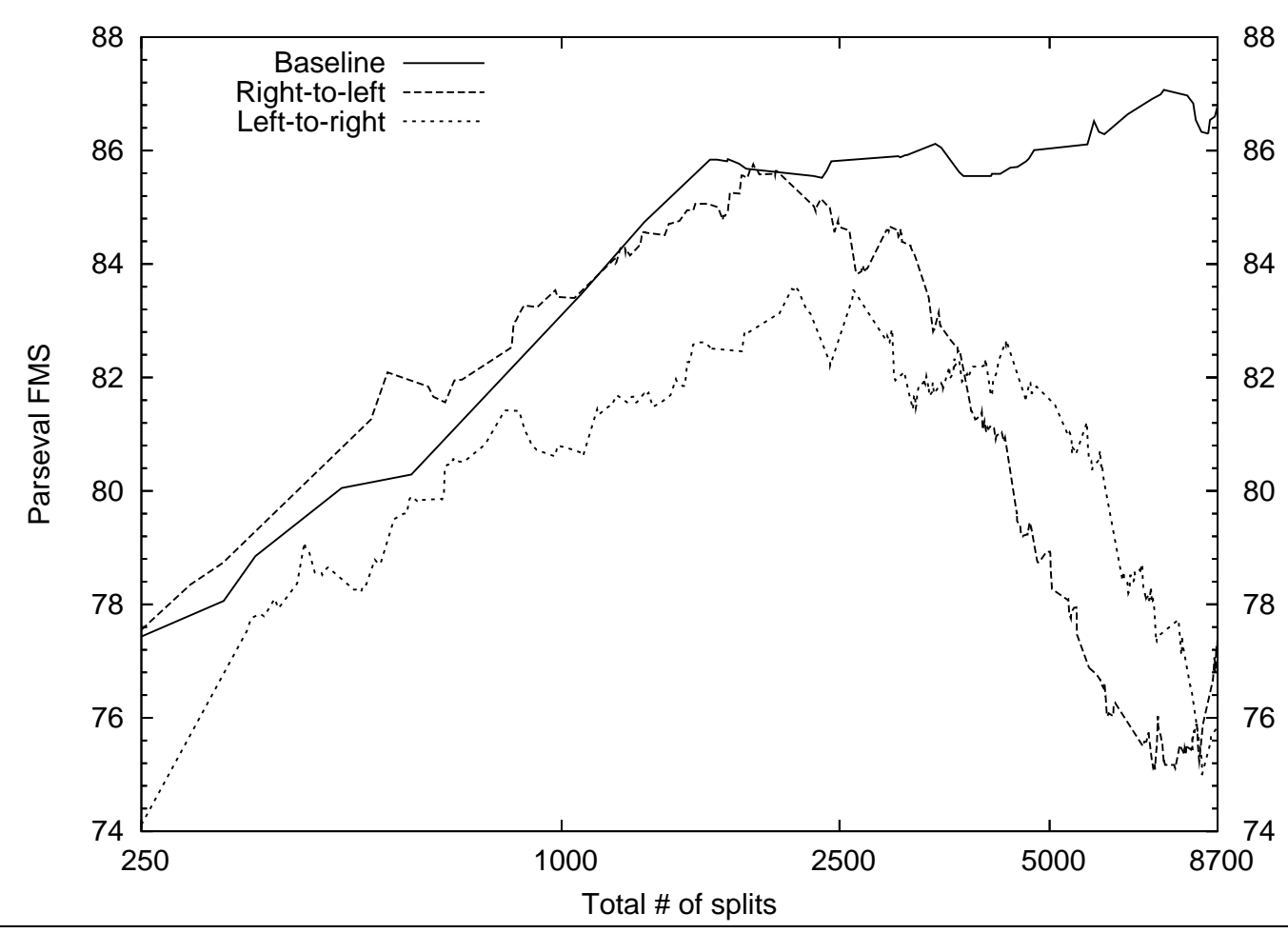

Figure 5 The margin distributions of the r2l NP-classifier, early in training and late in training, (a) over the incorrect training examples and (b) over the correct training examples.

(a)

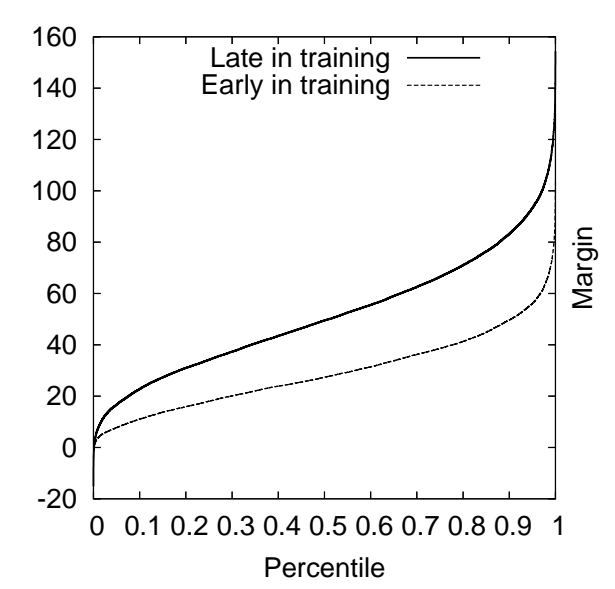

(b)

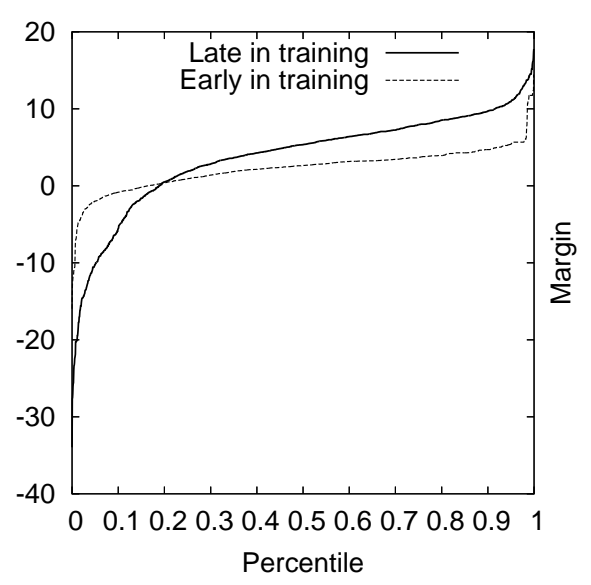

The amount of noise present in ADJP and ADVP annotations in the PTB is unusually high. Annotation of ADJP and ADVP unary projections is particularly inconsistent. For example, the development set contains the sentence "The dollar was trading sharply lower in Tokyo .", with "sharply lower" bracketed as "(ADVP (ADVP sharply) lower)". "sharply lower" appears 16 times in the complete training section, every time bracketed as "(ADVP sharply lower)", and "sharply higher" 10 times, always as "(ADVP sharply higher)". Because of the high number of negative examples, the classifiers' 
Table 9 PARSEVAL results of on the $\leq 15$ words test set of various parsers in the literature. The differences between the full vocabulary run and Bikel or Charniak are significant. Taskar et al. (2004)'s output was unavailable for significance testing, but presumably its differences from the full vocab parser are also significant.

$\begin{array}{lcccccc} & \text { Test } & \text { Test } & \text { Test } & \text { Dev } & \text { Dev } & \text { Dev } \\ & \text { LFMS } & \text { LRCL } & \text { LPRC } & \text { LFMS } & \text { LRCL } & \text { LPRC } \\ \text { Full vocab } & 87.13 & 86.47 & 87.80 & 87.50 & 86.85 & 88.15 \\ \text { Bikel (2004) } & 88.85 & 88.31 & 89.39 & 86.82 & 86.43 & 87.22 \\ \text { Taskar et al. (2004) } & 89.12 & 89.10 & 89.14 & 89.98 & 90.22 & 89.74 \\ \text { Charniak (2000) } & 90.09 & 90.01 & 90.17 & 89.50 & 89.69 & 89.32\end{array}$

bias is to cope with the noise by favoring negative confidences predictions for ambiguous ADJP and ADVP decisions, hence their abysmal labelled recall. One potential solution is the weight-sharing strategy described in Section 3.5.

- Tagging Errors Many of the parser's errors were due to poor tagging. Preprocessing sentence "Would service be voluntary or compulsory ?" gives "would/MD service/VB be/VB voluntary/J J or/CC UNK/J J" and, as a result, the parser brackets "service ...compulsory" as a VP instead of correctly bracketing "service" as an NP. We also found that the tagger we used has difficulties with completely capitalized words, and tends to tag them NNP. By giving the parser access to the same features used by taggers, especially rich lexical features (Toutanova et al., 2003), the parser might learn to compensate for tagging errors.

- Attachment decisions The parser does not detect affinities between certain word pairs, so it has difficulties with bilexical dependency decisions. In principle, bilexical dependencies can be represented as conjunctions of feature given in Section 2.1.4. Given more training data, the parser might learn these affinities.

\section{Conclusions}

In this work, we presented a near state-of-theart approach to constituency parsing which overcomes some of the limitations of other discriminative parsers. Like Yamada and Matsumoto (2003) and Sagae and Lavie (2005), our parser is driven by classifiers. Even though these classifiers themselves never do any parsing during training, they can be combined into an effective parser. We also presented a beam search method under the objective function of maximizing the minimum confidence.

To ensure efficiency, some discriminative parsers place stringent requirements on which types of features are permitted. Our approach requires no such restrictions and our scoring function can, in principle, use arbitrary information from the history to evaluate constituent inferences. Even though our features may be of too fine granularity to discriminate through linear combination, discriminatively trained decisions trees determine useful feature combinations automatically, so adding new features requires minimal human effort.

Training discriminative parsers is notoriously slow, especially if it requires generating examples by repeatedly parsing the treebank (Collins \& Roark, 2004; Taskar et al., 2004). Although training time is still a concern in our setup, the situation is ameliorated by generating training examples in advance and inducing one-vs-all classifiers in parallel, a technique similar in spirit to the POS-tag parallelization in Yamada and Matsumoto (2003) and Sagae and Lavie (2005).

This parser serves as a proof-of-concept, in that we have not fully exploited the possibilities of engineering intricate features or trying more complex search methods. Its flexibility offers many opportunities for improvement, which we leave to future work.

\section{Acknowledgments}

The authors would like to thank Dan Bikel, Mike Collins, Ralph Grishman, Adam Meyers, Mehryar Mohri, Satoshi Sekine, and Wei Wang, as well as the anonymous reviewers, for their helpful comments 
and constructive criticism. This research was sponsored by an NSF CAREER award, and by an equipment gift from Sun Microsystems.

\section{References}

Bikel, D. M. (2004). Intricacies of Collins' parsing model. Computational Linguistics, 30(4), 479-511.

Black, E., Abney, S., Flickenger, D., Gdaniec, C., Grishman, R., Harrison, P., et al. (1991). A procedure for quantitatively comparing the syntactic coverage of English grammars. In Speech and Natural Language (pp. 306-311).

Charniak, E. (2000). A maximum-entropy-inspired parser. In NAACL (pp. 132-139).

Cohen, P. R. (1995). Empirical methods for artificial intelligence. MIT Press.

Collins, M. (1999). Head-driven statistical models for natural language parsing. Unpublished doctoral dissertation, UPenn.

Collins, M. (2003). Head-driven statistical models for natural language parsing. Computational Linguistics, 29(4), 589-637.

Collins, M., \& Koo, T. (2005). Discriminative reranking for natural language parsing. Computational Linguistics, 31(1), 25-69.

Collins, M., \& Roark, B. (2004). Incremental parsing with the perceptron algorithm. In $A C L$.

Collins, M., Schapire, R. E., \& Singer, Y. (2002). Logistic regression, AdaBoost and Bregman distances. Machine Learning, 48(1-3), 253285.

Henderson, J. (2003). Inducing history representations for broad coverage statistical parsing. In HLT/NAACL.

Kalt, T. (2004). Induction of greedy controllers for deterministic treebank parsers. In EMNLP (pp. 17-24).

Kearns, M. J., \& Mansour, Y. (1999). On the boosting ability of top-down decision tree learning algorithms. Journal of Computer and Systems Sciences, 58(1), 109-128.

Klein, D., \& Manning, C. D. (2001). Parsing and hypergraphs. In IWPT (pp. 123-134).

Klein, D., \& Manning, C. D. (2003). Accurate unlexicalized parsing. In $A C L$ (pp. 423-430).
Magerman, D. M. (1995). Statistical decision-tree models for parsing. In ACL (pp. 276-283).

Marcus, M. P. (1980). Theory of syntactic recognition for natural languages. MIT Press.

Och, F. J., \& Ney, H. (2002). Discriminative training and maximum entropy models for statistical machine translation. In $A C L$.

Ratnaparkhi, A. (1996). A maximum entropy partof-speech tagger. In EMNLP (pp. 133-142).

Sagae, K., \& Lavie, A. (2005). A classifier-based parser with linear run-time complexity. In IWPT.

Schapire, R. E., \& Singer, Y. (1999). Improved boosting using confidence-rated predictions. Machine Learning, 37(3), 297-336.

Taskar, B., Klein, D., Collins, M., Koller, D., \& Manning, C. (2004). Max-margin parsing. In $E M N L P$ (pp. 1-8).

Taylor, A., Marcus, M., \& Santorini, B. (2003). The Penn Treebank: an overview. In A. Abeillé (Ed.), Treebanks: Building and using parsed corpora (pp. 5-22).

Toutanova, K., Klein, D., Manning, C. D., \& Singer, Y. (2003). Feature-rich part-of-speech tagging with a cyclic dependency network. In HLT/NAACL (pp. 252-259).

Wong, A., \& Wu, D. (1999). Learning a lightweight robust deterministic parser. In EUROSPEECH.

Yamada, H., \& Matsumoto, Y. (2003). Statistical dependency analysis with support vector machines. In IWPT.

Zhao, S., \& Grishman, R. (2005). Extracting relations with integrated information using kernel methods. In $A C L$. 